\title{
Resiliencia Familiar: un enfoque de investigación e intervención con familias multiproblemáticas
}

\author{
Family Resilience: a research and intervention approach \\ with multiproblem families
}

\author{
Esteban Gómez \\ María Angélica Kotliarenco ${ }^{2}$
}

\section{Resumen}

En este artículo se presenta el concepto resiliencia familiar, revisando sus antecedentes históricos, desarrollos actuales y posibles aplicaciones en el campo de la intervención clínica, psicosocial y de salud con familias altamente vulnerables. Este enfoque permite articular los aportes teóricos y empíricos de campos hasta ahora inconexos, como las ciencias del desarrollo, la terapia familiar y la intervención biopsicosocial con familias y niños vulnerables. Se distingue entre el riesgo crónico, la crisis significativa o la tensión familiar, en cuya presencia se activan procesos de resiliencia diferentes. La resiliencia familiar se define como el conjunto de procesos de reorganización de significados y comportamientos que activa una familia sometida a estrés para recuperar y mantener niveles óptimos de funcionamiento y bienestar, equilibrando sus recursos y necesidades familiares. Quedan numerosos aspectos metodológicos que resolver, como evaluar familias integralmente, la necesidad de estudios longitudinales y de investigación especialmente diseñada a partir de este enfoque.

Palabras Clave: resiliencia familiar, estrés familiar, crisis familiar, familias multiproblemáticas, intervención.

1 Esteban Gómez, Protectora de la Infancia, Psicólogo y Magíster en Psicología Clínica, Santiago de Chile, eagomez@uc.cl

2 María Angélica Kotliarenco, Centro de Estudios y Atención al Niño y la Mujer - CEANIM, Psicóloga, Master en Ciencias y Doctora en Filosofía, Santiago de Chile, mkotliarenco@gmail.com Dedicado en memoria de Ana María Haz, investigadora en maltrato infantil y resiliencia de la Escuela de Psicología de la Pontificia Universidad Católica, miembro fundador del Centro de Estudios de Emprendimientos Solidarios CEES-UC, mentora y amiga. 


\begin{abstract}
The concept of family resilience is presented, revising its historical antecedents, current developments and possible applications in the field of clinical, psychosocial and health intervention with highly vulnerable families. This approach allows the articulation of theoretical and empirical contributions of fields unconnected until now, such as developmental sciences, family therapy and biopsychosocial intervention with vulnerable families and children. It distinguishes between chronic risks, significant crisis or family tension, in whose presence different resilience processes are activated. Family resilience is defined as the group of processes of reorganization of meanings and behaviors, which a family under stress activates to recover and maintain optimal levels of functioning and wellbeing, balancing resources and family needs. There are a lot of methodological topics to be resolved, such as the integral evaluation of families, the need for longitudinal studies and research specially designed to be congruent with this approach.
\end{abstract}

Key Words: family resilience, family stress, family crisis, multiproblem families, intervention.

\title{
Introducción
}

El desarrollo puede entenderse como una trayectoria específica de intercambio entre los sistemas biológicos, psicológicos y sociales del ser humano, generando continuidad, discontinuidad y cambio en sus características, procesos y funciones a lo largo del ciclo vital (Bronfenbrenner \& Evans, 2000; McCartney \& Phillips, 2006). Desde su gestación y primera infancia hasta su muerte, la persona aborda tareas vitales y desafíos críticos para el logro de aprendizaje y competencia en las diversas áreas del desarrollo integral. Pero no está solo: se moldea y se construye a sí mismo en la relación y comunicación con otras personas, grupos, instituciones y referentes significativos de su cultura (Shonkoff\& Phillips, 2000). 
Desde un nivel genético, bioquímico y neuronal, el desarrollo humano se despliega en profunda articulación con lo interpersonal, vincular y socioafectivo, hasta lo cultural y colectivo (McCartney \& Phillips, 2006; Shonkoff \& Phillips, 2000). En ese camino, niños y adultos, familias y comunidades, deben lidiar con adversidades que ponen a prueba sus capacidades y recursos. Bajo ciertas condiciones, personas, familias y comunidades se ven limitadas, traumatizadas o incluso destruidas; por ejemplo, frente a una enfermedad crónica, maltrato grave, depresión materna, cesantía prolongada o catástrofes naturales. Hoy están científicamente documentados los efectos perjudiciales de estas experiencias adversas sobre el desarrollo humano (Petterson \& Burke, 2001; Shonkoff \& Phillips, 2000; Springer, Sheridan, Kuo, \& Carnes, 2007; Walker, Wachs, Gardner, Lozoff, Wasserman, Pollit et al., 2007); y sin embargo, nos siguen sorprendiendo historias de superación y resistencia, de transformación y crecimiento a partir del dolor y la adversidad.

Tras décadas de investigación en resiliencia, las respuestas a este fenómeno se buscan en la articulación de programas de investigación multidisciplinarios (Masten \& Obradovic, 2006). Este nuevo paradigma explica que en años recientes surgiese una renovada forma de mirar la resiliencia, ya no como una coraza personal de protección, sino como un engranaje relacional y eco-sistémico que permite encontrar oportunidades donde podría darse el estancamiento o deterioro.

El artículo busca profundizar esta idea desde el concepto de resiliencia familiar, que valoramos de utilidad para la intervención psicosocial, clínica y de salud con familias altamente vulnerables o multiproblemáticas. Se realizó una revisión temática usando las bases de datos EBSCO, ProQuest, Scielo y JSTOR, e ingresando: "resiliencia familiar", "estrés familiar", "factores de riesgo", "factores protectores", "adaptación familiar", "salud familiar" y "funcionamiento familiar", en español e inglés. 


\section{Antecedentes}

Reconociendo el enorme mérito de los pioneros de la investigación en resiliencia, es importante visualizar que los primeros intentos científicos llevaron a la hipótesis de la "invulnerabilidad" individual y a la imagen de "niños invencibles" (Werner \& Smith, 1982), haciéndose difícil visualizar la esencia relacional de los procesos de recuperación, sanación y crecimiento (Luthar, Cichetti \& Becker, 2000). Los primeros estudios sobre resiliencia la definieron como la capacidad de una persona para sobreponerse a la adversidad en forma competente (Werner \& Smith, 1982), centrándose en identificar factores de riesgo o condiciones de adversidad que aumentaban la probabilidad de un resultado negativo o patológico; historias individuales de adaptación exitosa a lo largo del ciclo vital; y factores protectores o condiciones que moderaban el efecto dañino de los factores de riesgo.

Estos estudios pioneros buscaban explicar por qué las condiciones de adversidad no llevaron, en esos niños, al resultado negativo esperado. Más tarde, se fue comprendiendo que la resiliencia no era una cualidad codificada en el código genético de la persona, ni que aplicase en todas las circunstancias ni en todos los dominios evaluados (Kalawski \& Haz, 2003). También se entendió que la resiliencia no es un fenómeno tan extraño y escaso como se creía inicialmente, sino que muchas veces forma parte de la vida cotidiana (Masten \& Obradovic, 2006). Se pasó entonces del estudio de lo extraordinario al estudio de lo cotidiano, y del estudio de la resiliencia como capacidad global, al estudio de las resiliencias múltiples, diferenciando cada contexto, grupo y problema particular (Kalawski \& Haz, 2003).

Recientemente, Masten \& Obradovic (2006) han expuesto una síntesis de los sistemas y procesos involucrados en el desarrollo humano exitoso frente a contextos de adversidad, según han sido reiteradamente identificados por las tres primeras generaciones de investigación en resiliencia, constituyendo las piedras angulares de un modelo eco-sistémico de resiliencia humana. Este conocimiento se grafica en la figura 1. 
Figura 1: Sistemas y procesos involucrados en la resiliencia frente a contextos de adversidad

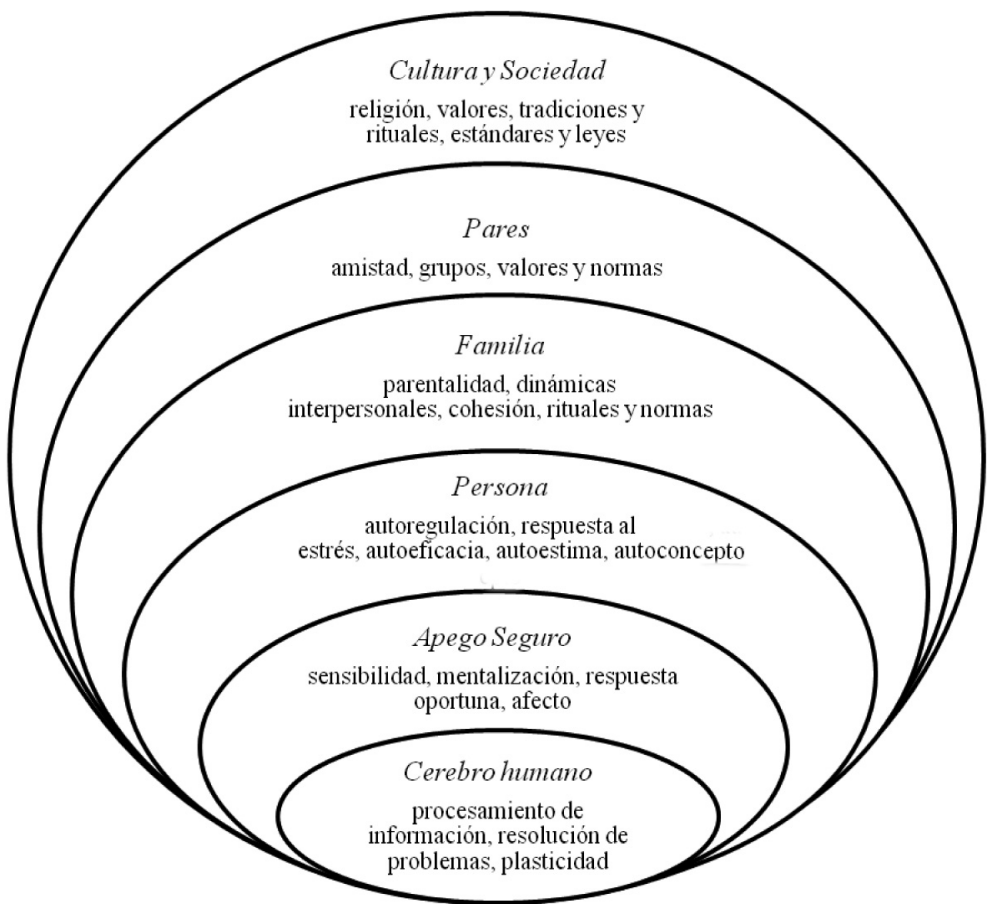

Fuente: construido desde Masten \& Obradovic, 2006.

Progresivamente, se esbozó un paradigma en el cual los lazos relacionales que unen a personas y sistemas les permiten caminar hacia una trayectoria compartida, encontrándose a la base de la mayoría de los procesos críticos para el desarrollo humano: por ejemplo, en los procesos biológicos y psicológicos del estrés, la autoregulación, la conformación de la citoarquitectura cerebral y su funcionamiento (Shore, 2001), en la construcción de modelos operativos o esquemas que organizan la experiencia y le confieren sentido (Fonagy \& Target, 1997), en la estabilidad dinámica que confiere salud mental (Lecannelier, 2009) y en la conquista de las condiciones biopsicosociales esenciales para el desarrollo (Walker et al., 2007), entre muchos otros. En todos ellos, las experiencias relacionales juegan un rol indesmentible (Shonkoff \& Phillips, 2000). 
Una ruta, dos caminos

Tanto a nivel individual como familiar el concepto resiliencia se concibe como una fuerza que se opone a la devastación potencial de la adversidad: no es posible hablar de resiliencia en ausencia de condiciones de adversidad con alta probabilidad de generar resultados negativos en una persona o grupo (Luthar et al. 2000; Masten \& Obradovic, 2006). La adversidad es entonces el germen de la resiliencia, el dolor es la semilla de la superación y los obstáculos son el incentivo al esfuerzo sostenido hacia una meta que caracteriza a las personas y familias resilientes (Cyrulnik, 2003).

La resiliencia, ya sea vista en personas o familias, no es una cualidad estática, un rasgo o característica inmutable, sino que es un proceso dinámico y cambiante que se manifiesta frente a ciertas exigencias, mientras que puede no observarse en otras condiciones o momentos (Kalawski \& Haz, 2003). Sin importar si es individual o familiar, la resiliencia es siempre contextual e histórica. Aunque este componente procesual de la resiliencia ha sido permanentemente enfatizado (De Haan, Hawley \& Deal, 2002; Rutter, 2007), continúa aplicándose en la práctica una búsqueda de aquella "cualidad intrínseca" a la persona, que explica sus resultados extraordinarios bajo estrés.

Otro aspecto compartido es que la resiliencia, a nivel personal o familiar, ha sido vista de dos formas. En un enfoque de resiliencia de "mínimos", se destaca el rol de la adaptación, es decir, la resiliencia sería un proceso dinámico de adaptaciones positivas dentro un contexto de significativa adversidad (Luthar et al., 2000; Rutter, 2007). Por otra parte, un enfoque de resiliencia de "máximos" no se limita a la recuperación o continuidad del equilibrio previo tras la crisis, sino que apuesta por la transformación y crecimiento. En esta mirada la resiliencia es la capacidad humana para enfrentar, sobreponerse y ser fortalecido o transformado por experiencias de adversidad (Cyrulnik, 2003).

Aunque comparten estos núcleos teóricos que dan cuerpo a la noción de resiliencia, la resiliencia individual tiene sus raíces en el estudio 
de la psicopatología evolutiva versus el desarrollo exitoso (Luthar et al., 2000), mientras que la resiliencia familiar se ancla en los estudios de terapia familiar y afrontamiento del estrés familiar (Kalil, 2003), mostrando un elevado potencial heurístico para la articulación teórica en la intervención clínica y psicosocial con familias altamente vulnerables (Gómez, Muñoz \& Haz, 2007; Sousa, 2008) y en el enriquecimiento de los nuevos modelos de atención en salud familiar (Schade, González, Beyebach, \& Torres, 2010).

Riesgo y Vulnerabilidad Familiar

Los factores de riesgo son variables que aumentan la probabilidad de un resultado disfuncional o patológico en un individuo o grupo (Rutter, 2007). En la investigación sobre resiliencia, el concepto "riesgo significativo" -fundamental al clarificar cuándo hablamos o no de resiliencia- dice relación con tres posibilidades (Masten \& Coatsworth, 1998, en Patterson, 2002b):

1. Exposición cotidiana y crónica a condiciones sociales adversas, como pobreza;

2. Exposición a un evento traumático, como abuso sexual, o una severa adversidad como la guerra o un terremoto;

3. Una combinación de alto nivel de riesgo con exposición a un evento traumático particular.

Por otra parte, el concepto factor de vulnerabilidad se refiere a variables que incrementan la susceptibilidad a los efectos negativos de los factores de riesgo, por ejemplo, una historia de depresión o baja cohesión familiar (Kalil, 2003). El modelo clásico del daño, entonces, señala que en presencia de un contexto adverso (múltiples factores de riesgo), si la persona o familia muestra determinadas debilidades en su funcionamiento (factores de vulnerabilidad) el impacto dañino de los problemas y crisis aumentará significativamente, pudiendo tornarse crónico de no mediar nuevos procesos de recuperación y fortalecimiento (ver figura 2). 
Figura 2: trayectorias múltiples de funcionamiento (deterioro v/s resiliencia) familiar

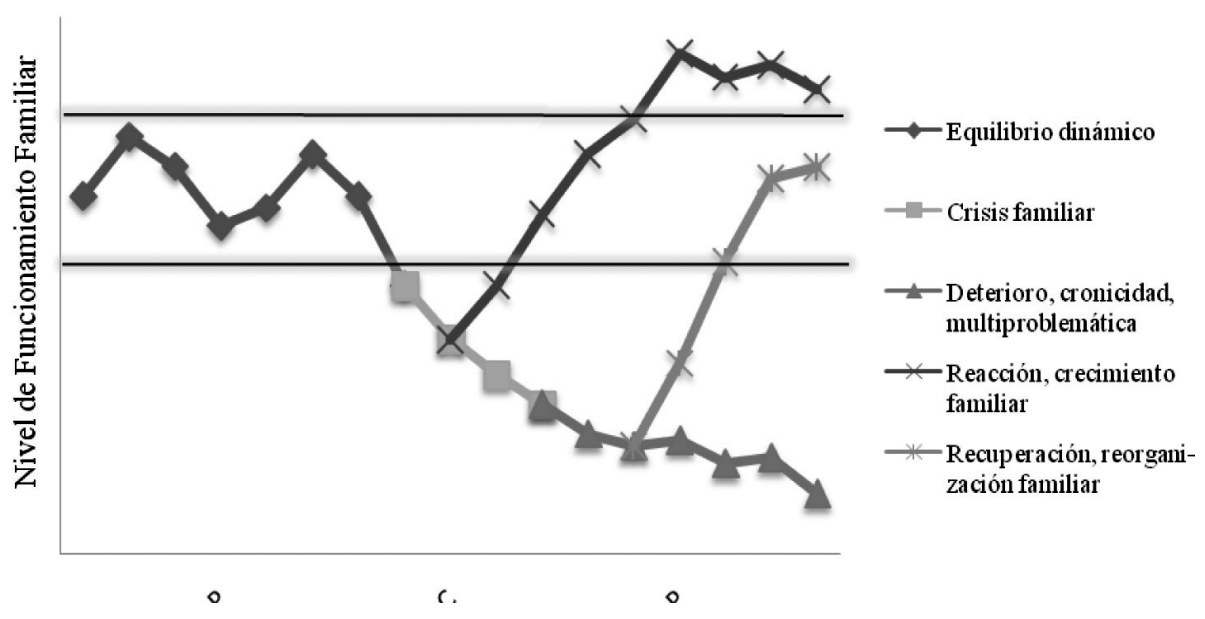

Fuente: elaboración propia.

Si bien estos procesos de riesgo y vulnerabilidad tocan a todas las familias a lo largo de su ciclo vital, existe un grupo en que se concentran con especial ahínco. Se trata de familias denominadas multiproblemáticas, caracterizadas por su polisintomatología, crisis recurrentes, más de un portador de síntomas, desorganización en su estructura y dinámica familiar, patrones de comunicación ambivalentes y empobrecidos, tendencia al abandono de sus funciones parentales, negligencia y maltrato infantil, y aislamiento o exclusión social (Gómez et al., 2007). Es decir, son familias donde la adversidad se ha tornado crónica y transgeneracional, y el involucramiento con agentes sociales es casi inevitable (Colapinto, 1995). Como estos servicios suelen caracterizarse por ser fragmentarios, reactivos a las crisis, centrados en el individuo y organizados a partir de los problemas y carencias (Gómez \& Haz, 2008; Sousa, 2008; Walsh, 2004), no es la resiliencia relacional lo que se estimula, sino más bien todo lo contrario, aquello que Colapinto (1995) ha denominado la disolución de los procesos familiares, entendida como la transferencia progresiva de las fortalezas y competencias de la familia hacia terceros, terminando en su eventual desintegración como sistema. 
Ahora bien, para actuar como catalizador de los procesos de cambio y crecimiento familiar no basta con adoptar simplemente una postura ingenua de "pensamiento positivo". El dolor, los traumas relacionales y las carencias agobiantes se encuentran presentes en una dimensión que el profesional percibe cada día y que resulta poco ético desconocer. Para recorrer el camino hacia la resiliencia familiar es indispensable entender el daño en una familia, y desde esa profunda empatía con su sufrimiento, avanzar en identificar sus puntos fuertes y procesos protectores, es decir, sus resortes relacionales hacia la superación.

Protección y Recuperación Familiar frente al estrés

El enfoque de la resiliencia familiar plantea que existen factores protectores cuya definición apunta a la función de "escudo" que ciertas variables cumplen sobre el funcionamiento familiar para mantenerlo saludable y competente bajo condiciones de estrés: por ejemplo, las celebraciones familiares, el tiempo compartido o las rutinas y tradiciones familiares. Estos factores actúan atenuando o incluso neutralizando el impacto de los factores de riesgo sobre la trayectoria evolutiva familiar (Kalil, 2003). En contrapunto, se identifican los procesos de recuperación, que cumplen una función de "catalizador" de nuevos equilibrios tras el impacto desestructurante de la crisis, por ejemplo, la comunicación, integración y apoyo familiar, la búsqueda de recreación y el optimismo familiar (Kalil, 2003; McCubbin \& McCubbin, 1988; Patterson, 2002b).

Tomando en cuenta estos elementos, la resiliencia familiar se ha definido como los patrones conductuales positivos y competencias funcionales que la unidad "familia" demuestra bajo estrés o circunstancias adversas, determinando su habilidad para recuperarse manteniendo su integridad como unidad, al tiempo que asegura y restaura el bienestar de cada miembro de la familia y de la familia como un todo (McCubbin, Balling, Possin, Frierdich \& Bryne, 2002).

Otra definición propone entender la resiliencia familiar como la descripción del camino que sigue una familia a medida que se adapta y prospera al afrontar el estrés, tanto en el presente como a lo largo del tiem- 
po. Las familias resilientes responden positivamente a estas condiciones específicas de adversidad en formas únicas, dependiendo del contexto, nivel de desarrollo, la combinación interactiva de factores protectores y de riesgo, y una visión familiar compartida (Hawley \& De Haan, 1996).

Las raíces de estas definiciones sobre resiliencia familiar pueden encontrarse en la Teoría sobre Desarrollo Familiar, que se formó a partir de la investigación sobre trayectorias familiares de afrontamiento del estrés (Gracia \& Musitú, 2000) desarrollada en la década de 1950 por Hill, en el modelo ABC$\mathrm{X}$. En dicho modelo, se identifican tres etapas posteriores a una crisis familiar:

1. Un período de desorganización: caracterizado por conflictos incrementales, búsqueda de formas de afrontamiento y una atmósfera de confusión, enojo y resentimiento;

2. Un período de recuperación: durante el cual se descubren nuevos medios para ajustarse a la crisis; $y$

3. Un período de reorganización: donde una familia se reconstruye hasta o sobre el nivel de funcionamiento anterior a la crisis.

Sin embargo, también es posible que un sistema familiar no se recupere del período de desorganización, llevando a su desintegración (De Haan et al., 2002). En la figura 2 se muestran trayectorias posibles de funcionamiento familiar antes, durante y posterior a la crisis.

En esta línea, uno de los principales desarrollos teóricos sobre resiliencia familiar proviene del trabajo de McCubbin, Patterson y colaboradores, quienes han explorado en una serie de estudios el comportamiento de las familias frente al estrés en el modelo Doble ABC-X (McCubbin \& Patterson, 1983; McCubbin \& McCubbin, 1988; McCubbin et al., 2002; Patterson, 1988; Patterson \& Garwick, 1994), desarrollando más recientemente un modelo de resiliencia familiar conocido como Respuesta Familiar de Ajuste y Adaptación (Family Adjustment and Adaptation Response - FAAR Model).

Este modelo de resiliencia familiar se levanta sobre cinco supuestos teóricos: las familias experimentan estrés y dificultades como un aspecto predecible de la vida familiar a lo largo del ciclo vital; poseen fortalezas y desarrollan competencias para proteger y asistir a sus miembros en la recuperación; se benefician y contribuyen a una red de relaciones en su co- 
munidad, particularmente durante períodos de estrés y crisis familiar; buscan, negocian y establecen una visión común, que les dará sentido, propósito y una perspectiva compartida para avanzar como grupo; y las familias enfrentadas a estrés y crisis significativas buscan restaurar el orden, balance y armonía incluso en medio de una etapa de gran conmoción (McCubbin et al., 2002).

En el modelo FAAR se enfatizan los procesos activos en los cuales las familias se involucran para equilibrar las demandas familiares con las capacidades familiares. Simultáneamente, este balance entre demandas y capacidades de la familia, interactúa con los significados familiares, para llegar a un nivel adecuado de ajuste o adaptación familiar (Patterson, 1988, 2002a, 2002b).

Las demandas familiares se componen de estrés normativo y no normativo, tensiones familiares constantes y complicaciones cotidianas, equiparándose a los factores de riesgo identificados en la literatura. Las capacidades familiares, por otra parte, incluyen los recursos tangibles y psicológicos (lo que la familia tiene) y los comportamientos de afrontamiento (lo que la familia hace), coincidiendo muchas veces con factores protectores y de recuperación. En cuanto a los significados familiares, se especifican significados situacionales sobre sus demandas y capacidades; su identidad como unidad familiar; y su visión de mundo, o cómo ven su familia en relación a otros sistemas.

De acuerdo al modelo, a partir de sus experiencias cotidianas, las familias se involucran en patrones estables de interacción, a medida que evalúan las demandas que enfrentan con las capacidades y recursos existentes, lo que las lleva a un nivel de ajuste familiar dinámico (ver figura 2). Sin embargo, en ciertos momentos las demandas familiares exceden significativamente sus capacidades, lo que produce un desequilibrio que, si persiste en el tiempo, desencadena una crisis familiar (Patterson, 2002a, 2002b). La resiliencia familiar se entiende como una recuperación del equilibrio, siendo compatible con el enfoque de la adaptación exitosa frente al estrés (Luthar et al., 2000; Rutter, 2007).

Se deriva, entonces, que los mecanismos para activar la resiliencia son: reducir las demandas familiares; aumentar sus capacidades; y/o cambiar los significados familiares. Estos procesos se denominan poder regenerativo o procesos de recuperación en el modelo FAAR (McCubbin $\&$ Patterson, 1983). 
Desde una perspectiva más clínica, Walsh (2003, 2004, 2007) ofrece un esquema que visualiza los procesos de resiliencia familiar en torno a tres ejes: los sistemas de creencias; los patrones organizacionales; y la comunicación y resolución de problemas en la familia (ver figura 3).

Figura 3: Procesos de la Resiliencia Familiar

\begin{tabular}{|ccc|}
\hline Proceso central & Componentes de cada & Caminos posibles a la \\
& proceso & resiliencia familiar
\end{tabular}

\begin{tabular}{|c|c|c|}
\hline & $\begin{array}{l}\text { Dar sentido a la } \\
\text { adversidad }\end{array}$ & $\begin{array}{l}\text { Ver la crisis como desafío } \\
\text { compartido }\end{array}$ \\
\hline \multirow[t]{3}{*}{ Creencias } & $\begin{array}{c}\text { Perspectiva positiva y } \\
\text { esperanza }\end{array}$ & $\begin{array}{l}\text { Focalizar en fortalezas, } \\
\text { dominar lo posible }\end{array}$ \\
\hline & $\begin{array}{l}\text { Trascendencia y } \\
\text { espiritualidad }\end{array}$ & Aprender y crecer \\
\hline & $\begin{array}{c}\text { Flexibilidad, cambio, } \\
\text { estabilidad }\end{array}$ & $\begin{array}{l}\text { Recuperarse, } \\
\text { reorganizarse }\end{array}$ \\
\hline \multirow[t]{3}{*}{ Organización } & Conexión /cohesión & $\begin{array}{l}\text { Apoyo mutuo, respeto, } \\
\text { reconciliarse }\end{array}$ \\
\hline & Recursos sociales & $\begin{array}{l}\text { Movilizar redes, lograr } \\
\text { seguridad económica }\end{array}$ \\
\hline & Claridad & $\begin{array}{l}\text { Buscar coherencia entre } \\
\text { palabras y acciones }\end{array}$ \\
\hline \multirow[t]{2}{*}{ Comunicación } & $\begin{array}{l}\text { Expresión sincera y } \\
\text { empatía }\end{array}$ & $\begin{array}{l}\text { Evitar acusaciones, } \\
\text { compartir experiencias }\end{array}$ \\
\hline & $\begin{array}{l}\text { Resolución } \\
\text { problemas } \\
\text { colaborativa }\end{array}$ & $\begin{array}{l}\text { Medidas concretas, ir } \\
\text { paso a paso, prevenir }\end{array}$ \\
\hline
\end{tabular}

Fuente: construido desde Walsh, 2003, 2004, 2007. 
Sistema de creencias compartido

De acuerdo a este modelo, las familias resilientes logran construir un sistema de creencias compartido que las orienta hacia la recuperación y el crecimiento. Este primer proceso de la resiliencia familiar se torna posible al normalizar y contextualizar la adversidad y el estrés, generando un sentido de coherencia que redefine la crisis como un desafío manejable. Este concepto también ha recibido el nombre de "esquema familiar" en la literatura sobre resiliencia familiar (Hawley, 2000).

En las familias que logran activar su resiliencia se observa un concepto evolutivo del tiempo y del devenir, como un proceso continuo de crecimiento y cambio; en contraste, las familias que se estancan en patrones disfuncionales -especialmente las multiproblemáticas- carecen de este sentido y sus síntomas suelen aparecer en momentos de transición disruptiva, que las congelan y angustian (Coletti \& Linares, 1997).

Para hacer surgir la resiliencia familiar se requiere que la familia mantenga una visión positiva, pero realista de la situación, dominando lo posible y aceptando lo inevitable. En este proceso juega un rol importante la trascendencia y la espiritualidad, aspectos que hasta hace poco eran considerados ajenos a la investigación científica. El impulso a trascender inspira a vislumbrar nuevas posibilidades, encontrando muchas veces en la fe el motor para el crecimiento a partir del golpe de la crisis.

\section{Patrones organizacionales}

En segundo lugar, el modelo propuesto por Walsh $(2003,2004)$ destaca la fuerza protectora de los patrones organizacionales de la familia, que actúan como absorbentes de las conmociones familiares. En estos patrones se encuentra la movilidad versus estancamiento de una familia en crisis; ya que la crisis tiene el potencial para desestructurar las formas conocidas de funcionamiento previo, la flexibilidad emerge como un elemento central. La flexibilidad o plasticidad familiar, como símil de la plasticidad neuronal, conlleva la capacidad de abrirse al cambio, de 
reorganizar el entramado de posiciones y roles de cada componente del sistema para adaptarse a nuevos desafíos. Sin embargo, la forma específica en que esto se resuelva no es única ni excluyente: hay muchas formas posibles de organización en una familia y cultura determinadas (Gracia \& Musitu, 2000).

La capacidad de reorganización familiar tras la crisis se sustenta en lo que Walsh $(2003,2004)$ denomina conexión familiar, pero que Olson (1989, en Kalil, 2003) y otros han nombrado cohesión familiar. Implica apoyo mutuo y compromiso hacia metas colectivas. Cuando se intenta estimular la conexión familiar, las viejas rencillas, los "fantasmas del pasado", dificultan notoriamente este proceso, siendo por tanto necesario buscar la reconciliación en las relaciones dañadas, el perdón, o al menos una tregua temporal.

Parte de los procesos de reorganización familiar frente a una crisis consisten en incorporar nuevos recursos en forma coordinada (Landau, 2007). Ahora bien, la búsqueda de nuevos recursos de apoyo conlleva el riesgo paradojal de abrir demasiado el sistema familiar a la intervención de terceros, quienes pueden involucrarse excesivamente, en forma descoordinada y poco sensible al ethos familiar, terminando por desorganizar y disolver sus procesos familiares de autonomía y resiliencia (Colapinto, 1995). Este riesgo nunca debe ser subestimado, y en toda intervención la pregunta por la pertinencia, articulación y dosificación de las acciones juega un rol central.

Comunicación y resolución de problemas

En tercer lugar, los procesos de resiliencia familiar se sustentan en la comunicación y habilidades para la resolución de problemas. Esta variable ha sido muy trabajada por los terapeutas familiares (Hawley, 2000), quienes han concordado en que debe ser clara, favorecer la expresión emocional abierta y la búsqueda colaborativa de soluciones (Minuchin 
\& Fishman, 2004; Navarro Góngora \& Beyebach, 1995). Asimismo, se requiere que los miembros de la familia puedan compartir un amplio rango de emociones, como alegría y dolor, esperanzas y temores, éxitos y frustraciones (Minuchin \& Fishman, 2004; Navarro Góngora \& Beyebach, 1995; Walsh, 2003).

Las familias multiproblemáticas han sido "entrenadas" por su historia y su relación con los agentes sociales para destacar lo que no funciona (Colapinto, 1995; Coletti \& Linares, 1997; Sousa, 2008), lo que sale mal o bajo las expectativas (sin considerar que a veces son expectativas inaplicables), y muy poco para iluminar y celebrar lo que si funciona. Precisamente la literatura sobre efectividad de terapia familiar (Minuchin \& Fishman, 2004) y de intervenciones psicosociales con familias multiproblemáticas (Sousa, 2008) ha respaldado el esquema de definir pasos concretos hacia una meta co-construida, avanzando sobre el fundamento de las pequeñas conquistas personales y colectivas.

Hallazgos empíricos sobre resiliencia familiar

Si bien existe poca investigación desarrollada específicamente desde el marco teórico de la resiliencia familiar (De Haan et al., 2002), comienzan a visualizarse hallazgos congruentes que apuntan hacia una dirección común.

Las investigaciones cualitativas han mostrado procesos específicos de resiliencia familiar según el contexto abordado, pero también procesos transversales. En la Tabla 1 se ilustra este punto con tres estudios: adversidad psicosocial y crianza positiva, desplazamiento familiar por violencia armada, y enfermedad de cáncer en un hijo. Los procesos reiterados fueron: la perseverancia en objetivos y actividades que beneficiaron la unidad familiar; el soporte o apoyo mutuo mediante una actitud empática con el otro; y la búsqueda activa de nuevas redes de apoyo. 
Tabla 1: Estudios cualitativos sobre resiliencia familiar

\begin{tabular}{|c|c|c|c|}
\hline Contexto & $\begin{array}{l}\text { Adversidad psicosocial y } \\
\text { crianza positiva } \\
\text { (Silva et al., 2009; estudio } \\
\text { de caso) }\end{array}$ & $\begin{array}{l}\text { Desplazamiento familiar } \\
\text { por violencia armada } \\
\text { (González, 2004; } 9 \\
\text { familias entrevistadas) }\end{array}$ & $\begin{array}{l}\text { Cáncer en un hijo } \\
\text { (McCubbin et al., } \\
\text { 2002; } 26 \text { familias y } 42 \\
\text { entrevistas) }\end{array}$ \\
\hline $\begin{array}{l}\text { Procesos } \\
\text { específicos }\end{array}$ & $\begin{array}{ll}\checkmark & \text { sensibilidad parental } \\
\checkmark & \text { responder a las } \\
\text { necesidades de sus hijos } \\
\checkmark \quad \text { crear un espacio } \\
\text { relacional de expresión del } \\
\text { potencial de los hijos }\end{array}$ & $\begin{array}{l}\checkmark \quad \text { la vida como valor } \\
\checkmark \quad \text { generar nuevos } \\
\text { proyectos de vida } \\
\checkmark \quad \text { mantener la unión } \\
\text { familiar a distancia } \\
\checkmark \quad \text { resolución } \\
\text { conjunta de problemas } \\
\checkmark \quad \text { buen humor } \\
\text { como estrategia de } \\
\text { comunicación } \\
\checkmark \quad \text { generosidad }\end{array}$ & $\begin{array}{l}\checkmark \quad \text { rápida } \\
\text { movilización y } \\
\text { reorganización familiar } \\
\checkmark \quad \text { apoyo social } \\
\text { del equipo de salud, } \\
\text { familia extensa, } \\
\text { comunidad y lugar de } \\
\text { trabajo } \\
\checkmark \quad \text { cambios en la } \\
\text { evaluación de su } \\
\text { experiencia } \\
\checkmark \quad \text { hacer la situación } \\
\text { más comprensible, } \\
\text { manejable y con } \\
\text { sentido }\end{array}$ \\
\hline $\begin{array}{l}\text { Procesos } \\
\text { comunes }\end{array}$ & \multicolumn{3}{|c|}{$\begin{array}{l}\text { perseverancia en objetivos y actividades que beneficien la unidad familiar } \\
\text { soporte mutuo mediante una actitud empática } \\
\text { aceptar ayuda en el rol; construir nuevas redes de apoyo }\end{array}$} \\
\hline
\end{tabular}

Asimismo, los estudios cuantitativos expuestos en la Tabla 2 reafirman la diferenciación entre procesos específicos al contexto, versus procesos transversales, destacando entre estos últimos: generar espacios y procesos de comunicación en la familia; resolver conjuntamente los problemas; fortalecer la cohesión familiar y apoyo mutuo; enriquecer la calidad de la interacción padre-hijo; practicar y desarrollar las competencias parentales; y acceder a fuentes de apoyo social (Benzies \& Mychasiuk, 2008). 


\section{Tabla 2: Estudios cuantitativos sobre resiliencia familiar}

\begin{tabular}{|c|c|}
\hline Contexto del estudio & Hallazgos principales sobre procesos de resiliencia familiar \\
\hline $\begin{array}{l}\text { Familias en riesgo psicosocial } \\
\text { (Rodrigo et al., 2009; } \mathrm{N}=418 \text { ) }\end{array}$ & $\begin{array}{l}\checkmark \text { la resiliencia no está determinada por factores } \\
\text { evolutivos o estructurales. } \\
\checkmark \quad \text { depende de las oportunidades que brindan los } \\
\text { contextos de desarrollo. } \\
\checkmark \quad \text { resulta clave favorecer el desarrollo de las competencias } \\
\text { parentales. }\end{array}$ \\
\hline $\begin{array}{l}\text { Familias con una hospitalización } \\
\text { psiquiátrica } \\
\text { (Johnson, 1998; N=180) }\end{array}$ & $\begin{array}{ll}\checkmark & \text { nivel de funcionamiento familiar; y } \\
\checkmark & \text { sentido familiar de competencia. }\end{array}$ \\
\hline $\begin{array}{l}\text { Familias con escasos ingresos } \\
\text { económicos } \\
\text { (Orthner et al., 2004; N=373) }\end{array}$ & $\begin{array}{ll}\checkmark & \text { comunicación familiar; } \\
\checkmark & \text { resolución de problemas; } \\
\checkmark & \text { acceso al apoyo social. }\end{array}$ \\
\hline $\begin{array}{l}\text { Familias a lo largo del ciclo vital } \\
\text { (McCubbin \& McCubbin, 1998; } \\
\mathrm{N}=360 \text { ) }\end{array}$ & $\begin{array}{l}\checkmark \text { comunicación para intercambiar información y } \\
\text { cuidados; } \\
\checkmark \text { acuerdo para resolver conflictos; } \\
\checkmark \text { "fortaleza" como sentido de control, compromiso con } \\
\text { la familia y confianza; } \\
\checkmark \text { aceptación del otro; } \\
\checkmark \quad \text { tiempo y rutinas que permitan continuidad y } \\
\text { estabilidad en la vida familiar; celebraciones; actividades } \\
\text { de ocio compartidas; } \\
\checkmark \text { tradiciones para honrar la historia y experiencias } \\
\text { familiares; } \\
\checkmark \text { manejo financiero; } \\
\checkmark \text { salud física y psicológica; } \\
\checkmark \text { red de apoyo positiva (parientes, cercanos y amigos). }\end{array}$ \\
\hline $\begin{array}{l}\text { Familias con un hijo autista } \\
\text { (Greeff \& van der Walt, 2010; } \\
\mathrm{N}=34)\end{array}$ & $\begin{array}{ll}\checkmark & \text { patrones de comunicación abiertos y predecibles; } \\
\checkmark & \text { entorno familiar de apoyo, comprometido y flexible; } \\
\checkmark & \text { "fortaleza" familiar; } \\
\checkmark & \text { estrategias de afrontamiento internas y externas; } \\
\checkmark & \text { apoyo social; } \\
\checkmark & \text { estatus socioeconómico; } \\
\checkmark & \text { una mirada positiva de la vida; } \\
\checkmark & \text { y el sistema de creencias familiares. }\end{array}$ \\
\hline
\end{tabular}


Como puede verse, la investigación empírica cualitativa y cuantitativa ha identificado procesos de resiliencia familiar altamente consistentes con los modelos teóricos reseñados en este artículo. Sin embargo, se requiere mucha más investigación desarrollada específicamente desde este enfoque, lo que implica ciertos desafíos metodológicos que se analizarán en la discusión.

Aplicaciones posibles del enfoque de resiliencia familiar

El concepto de resiliencia familiar ha sido aplicado recientemente en la terapia familiar (Hawley, 2000; Walsh, 2003), en los programas integrales de intervención clínica familiar (Lee, Greene, Hsu, Solovey, Grove, Fraser et al., 2009), en intervención psicosocial con familias multiproblemáticas (Sousa, 2008), en servicios de terapia ocupacional y salud mental (Abelenda \& Helfrich, 2003), en el campo de la salud familiar (Borges \& Silva, 2010) y en el manejo de catástrofes naturales, terrorismo o crisis económicas (Landau, 2007, 2010; Walsh, 2007).

A continuación se muestran iniciativas implementadas en Chile que se consideran potencialmente congruentes con los principios de un modelo ecosistémico de resiliencia familiar, en tres áreas de alta relevancia pública: la promoción del desarrollo infantil temprano; la prevención secundaria del maltrato infantil y la negligencia parental; y la atención en centros de salud familiar.

Los Centros de Desarrollo Infantil Temprano para el apoyo familiar desde el embarazo hasta el segundo ańo de vida del infante fue un proyecto implementado por el Centro de Estudios y Atención al Niño y la Mujer (CEANIM) entre 2006 y 2008 en un total de 15 centros y 829 familias. El proyecto se concentró en fortalecer la díada madre-hijo desde el embarazo hasta el segundo año, en mujeres de bajo nivel socio-económico, viviendo en condiciones de precariedad, riesgo psicosocial y exclusión de las redes locales de apoyo. La principal estrategia fue la visita domiciliaria intensiva, complementada con consejerías, talleres psicoeducativos y cuidado temporal del bebé. La evaluación de resultados pre-post (Kotliarenco, Muñoz, Gómez, \& Armijo, 2009), mostró una mejoría estadísticamente significativa en el desarrollo psicomotor de los infantes, observándose un 
efecto preventivo para casos con normalidad inicial y nivelador para casos en riesgo inicial $(N=166, p<0.05)$. Además, se constató un aumento en la clasificación de apego seguro y disminución del apego evitativo $(N=49$, $p<0.01)$. Este proyecto dio origen a un nuevo piloto actualmente en curso, denominado Centro de Buenas Prácticas en Infancia Temprana.

Viviendo en Familia es un programa para la prevención secundaria del maltrato infantil y la negligencia parental, implementado por la Protectora de la Infancia en 11 centros con un total de 900 nińos y sus familias. Un primer estudio demostró una adecuada focalización en su población objetivo, acogiendo familias con elevados niveles de estrés, riesgo y vulnerabilidad (Gómez, Cifuentes \& Ross, 2010). El programa fue diseñado articulando el marco de la resiliencia y la teoría eco-sistémica del maltrato infantil; busca revertir la trayectoria de deterioro familiar, activando sus recursos, competencias y procesos de recuperación; disminuyendo los niveles de tensión y estrés familiar; y aumentando sus niveles de buen trato, protección y autonomía. Usando la Escala de Evaluación Familiar (NCFAS), se evalúan dificultades y fortalezas en cinco áreas: entorno, competencias parentales, interacciones familiares, protección familiar y bienestar del niño (Valencia \& Gómez, 2010) y el plan de trabajo se co-construye con la familia y redes significativas. Los servicios ofrecidos son consejerías, visitas domiciliarias, terapia breve, talleres y articulación de la red de apoyo. Una evaluación de proceso dio cuenta de la correcta implementación del modelo, logrando una valoración positiva por todos los actores involucrados. La evaluación de resultados (Gómez \& Cifuentes, 2010), mostró que en sus tres primeros años de funcionamiento se egresó exitosamente al $73 \%$ de los casos. En el foco de maltrato infantil y negligencia parental, aumentó el funcionamiento "adecuado/fortalecido" desde un $25 \%$ inicial hasta un $75 \%$ final; se constató una mejoría estadísticamente significativa en todas las dimensiones del funcionamiento familiar evaluadas con la NCFAS $(N=580 ; p<.0001)$, con magnitudes de la diferencia pre-post grandes -sobre 0.8- según los criterios de Cohen.

Recientemente, se implementó un modelo de consejería familiar, denominado Abordaje Familiar Breve, evaluando sus resultados en 128 
pacientes con trastornos somatomorfos atendidos en siete Centros de Salud Familiar de la red de atención primaria, región del Bio-Bío (Schade et al., 2010). El modelo recoge las premisas del enfoque de salud familiar, trabajando con el supuesto de que los pacientes tienen recursos y fuerzas suficientes para resolver sus problemas. Se plantean tres etapas: preconsejería familiar en salud o interiorización acerca del caso; la consejería familiar, con cuatro fases: co-construcción de la relación; reflexión y análisis para la toma de decisiones; abordaje orientativo, informativo y estratégico; y negociación, tarea y contrato; y post-consejería familiar, o evaluación; y seguimiento de los cambios. Es interesante que aunque el diagnóstico de ingreso era trastorno somatomorfo, la queja principal reportada por los pacientes decía relación con la pareja y familia en un 58,5\%. Las sesiones variaron entre 1 y 5 , con un promedio de 3 , y se obtuvieron resultados positivos en el 75,8\% de los casos.

Aunque en ninguna de estas experiencias pueda realizarse atribuciones causales por falta de un grupo de control, sus resultados positivos son estimulantes respecto al potencial de este tipo de programas en el país. Se consideran ejemplos del tipo de iniciativas diseñadas e implementadas en Chile que resultan coherentes con el marco de la resiliencia familiar. Las tres experiencias descritas enfatizan los recursos y potencialidades de cambio y crecimiento de las familias atendidas, por sobre el déficit y la patología; se articulan con el contexto ecológico de desarrollo de la familia; y trabajan activamente por construir una relación respetuosa y colaborativa con las familias.

\section{Discusión}

El enfoque de la resiliencia familiar muestra un futuro prometedor y desafiante. Bajo este concepto, una gran cantidad de investigación y desarrollo técnico puede ser articulado en un todo coherente que permita profundizar el conocimiento sobre el funcionamiento familiar saludable y patológico en el ciclo vital, mejorando significativamente el impacto de las intervenciones, especialmente con familias vulnerables que presenten diversos grados de multi-problematicidad. 
Como se vio, el enfoque de la resiliencia familiar se nutre de la resiliencia individual, en especial del esquema que tensiona factores de riesgo y vulnerabilidad versus factores protectores y de recuperación. Pero a su vez ha desarrollado conceptos específicos, al entender la crisis como un desequilibrio entre demandas, capacidades y significados construidos por la familia (Patterson, 2002a, 2002b), o la visualización de los sistemas de creencias, procesos organizacionales y patrones de comunicación y resolución de problemas, como procesos articuladores de la resiliencia familiar (Walsh, 2003, 2004, 2007).

La figura 4 es un esquema que busca integrar los diversos tópicos revisados en un Modelo Ecosistémico de Resiliencia Familiar. El esquema muestra que el desarrollo individual y familiar ocurre en la interacción e intercambio de recursos tangibles e intangibles entre diversos sub-sistemas y eco-sistemas anidados (Gracia \& Musitu, 2000; McCartney \& Phillips, 2006; Shonkoff \& Phillips, 2000).

Figura 4: Modelo Eco-Sistémico de Resiliencia Familiar

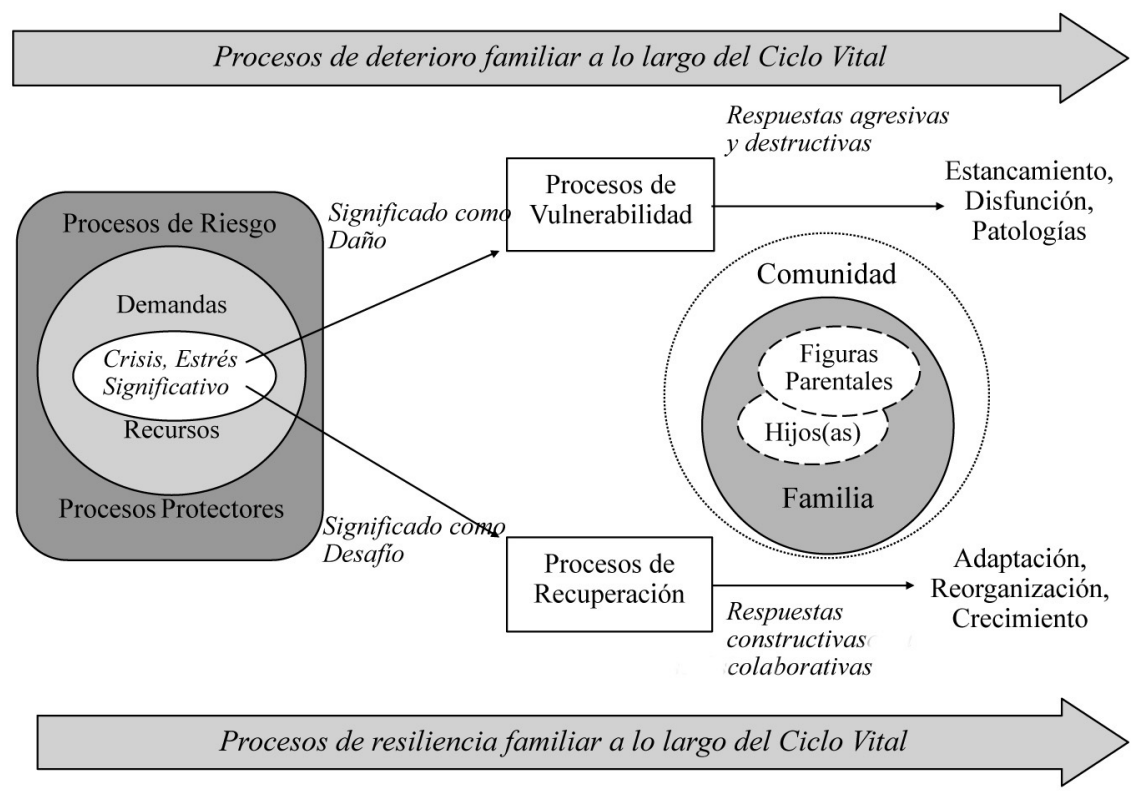

Fuente: elaboración propia. 
Se enfatiza el rol crítico que juegan los procesos de significación intersubjetiva construidos por la familia durante períodos de estrés significativo o en etapas posteriores a la crisis, generando trayectorias familiares diferenciadas: aquellas del estancamiento o bien del deterioro progresivo de la organización, funciones y salud familiar (que incluso entonces pueden revertirse con una adecuada intervención), o bien aquellas trayectorias que activan la resiliencia familiar.

Proponemos que la resiliencia familiar se defina como el conjunto de procesos de reorganización de significados y comportamientos que activa una familia sometida a estrés, para recuperar y mantener niveles óptimos de funcionamiento y bienestar, equilibrar recursos y necesidades familiares, y aprovechar las oportunidades de su entorno. Identificamos tres escenarios posibles para la activación o estancamiento de la resiliencia familiar (ver figuras 2 y 4$)$ :

- Un contexto de alto riesgo biopsicosocial, equilibrado por procesos protectores activos, es decir, una resiliencia familiar por contrapeso cotidiano: por ejemplo, una familia vive en un barrio pobre, con altos niveles de violencia y microtráfico, pero logra mantener valores prosociales, roles familiares flexibles y rutinas compartidas, contrapesando las influencias potencialmente dañinas del entorno.

- Una crisis en que los factores de riesgo, vulnerabilidad y estrés (ej., cesantía, depresión parental y alcoholismo) superan los recursos familiares, generando un fuerte deterioro en la familia (ej., dinámicas de maltrato infantil); tras lo cual se activan procesos relacionales de superación (ej., la familia conversa su problema, es más probable en etapas tempranas del proceso) o bien requiere la intervención de terceros (ej., ingresa a un programa de intervención familiar, en etapas más avanzadas del deterioro). Es una resiliencia familiar por recuperación.

- El tercer escenario identifica un evento con alto nivel de exigencia y estrés (ej., un diagnóstico de grave enfermedad), pero no se genera un proceso de deterioro familiar prolongado, activándose prontamente el capital acumulado por la familia (ej., una espiritualidad compartida durante años) o la movilización de recursos externos (ej., apoyo profesional 
inmediato), generando un funcionamiento incluso superior, es decir, una resiliencia familiar por crecimiento.

Esto muestra que podemos estimular la resiliencia familiar desde nuestro rol profesional, ya sea: disminuyendo las demandas y tensiones que sobrepasan a la familia, facilitando su capacidad de contrapeso; promoviendo y movilizando sus capacidades, fortalezas y recursos disponibles -en ellas mismas y en su entorno-; estimulando la cohesión o conexión relacional entre sus miembros; y elaborando y modificando sus sistemas de creencias y significados compartidos (Hawley, 2000; Kalil, 2003; Landau, 2007, 2010; Patterson, 2002b; Sousa, 2008; Walsh, 2003, 2007).

Es importante, en el análisis previo a comenzar la intervención, darse tiempo para identificar claramente el escenario que se enfrenta (riesgo, deterioro o tensión familiar) para seleccionar la estrategia adecuada a seguir (contrapeso, recuperación o fortalecimiento preventivo). En esa evaluación es fundamental contar con herramientas que faciliten una visión integral de la familia, tanto de sus problemas como fortalezas, en concordancia con un enfoque ecológico de la resiliencia.

Existen múltiples aplicaciones potenciales de la teoría sobre resiliencia familiar, en campos tan diversos como la psicoterapia, los programas psicosociales, los centros de salud familiar en atención primaria, los programas de infancia temprana y los centros de educación preescolar y escolar. La resiliencia como enfoque hoy es patrimonio de la humanidad, siendo el estandarte que orienta las iniciativas gubernamentales dirigidas a la infancia vulnerable o a procesos de reconstrucción posteriores a catástrofes naturales. Todas estas aplicaciones sin duda han contribuido a extender y dar visibilidad al concepto y su marco teórico entre la población general, permitiendo que se apoye la investigación y el desarrollo de nuevos programas de intervención.

Sin embargo, la aplicación sistemática basada en evidencia sobre resiliencia ha sido mucho más la excepción que la norma. Existen importantes diferencias entre cómo se conceptualiza metodológicamente la resiliencia y cómo la entienden los practicantes que aplican el concepto (Patterson, 2002b). Generalmente, los practicantes usan el concepto para referirse a un enfoque que destaca las fortalezas de la familia por sobre sus 
defectos (Sousa, 2008); la mayoría de los investigadores, en cambio, han estado más interesados en los resultados demostrados frente al riesgo (De Haan et al., 2002).

Por ello, se requiere desarrollar investigación que sea concebida específicamente bajo este marco conceptual, surgiendo numerosas dificultades metodológicas a tomar en cuenta. Por ejemplo, la disyuntiva entre evaluar familias como unidades integrales, en contraposición a recoger una colección de percepciones de individuos. Si bien existen procedimientos para manejar datos recolectados individualmente (sumar puntajes, obtener promedios, etc.), permanece el problema de que los procesos familiares pertenecen a un nivel distinto y debiesen ser operacionalizados y evaluados en dicho nivel (Cumsille, 1996; De Haan et al., 2002).

Los diseños de investigación en resiliencia familiar debiesen ser longitudinales, incluir mediciones anteriores y posteriores a una crisis y recolectar información a nivel familiar (no únicamente individual); asimismo, deben ser sensibles al contexto de la familia y a los estresores examinados. Al respecto, Patterson (2002a) realiza las siguientes propuestas para el avance de la investigación en resiliencia familiar:

- Ofrecer claras definiciones operacionales y conceptuales de variables clave, diferenciando los resultados que indican competencia familiar, de los procesos protectores familiares activados.

- Desarrollar y testear modelos conceptuales sobre procesos familiares protectores y de riesgo.

- Estudiar poblaciones de familias experimentando riesgos significativos (violencia de pareja, maltrato infantil, cesantía, etc.) y no solo crisis normativas.

- Conducir estudios longitudinales.

- Incorporar métodos cualitativos en la investigación, con especial énfasis en los procesos de construcción de significado en las familias.

En este desafío, creemos que instrumentos como la Escala de Evaluación Familiar NCFAS aportan nuevas herramientas para diseñar estudios de cuarta generación sobre resiliencia familiar (Valencia \& Gómez, 2010). Asimismo, el diseño e implementación de nuevos modelos de apoyo e intervención familiar y comunitaria, especialmente frente a 
familias multiproblemáticas y en riesgo social (Gómez \& Cifuentes, 2010; Kotliarenco et al., 2009; Landau, 2007, 2010; Lee et al., 2009; Sousa, 2008) requieren potenciar progresivamente el uso de las herramientas que este enfoque ofrece.

$\mathrm{Al}$ abrir la puerta al estudio, conceptualización e intervención de la resiliencia relacional (grupal, familiar o comunitaria) se han abierto desafíos de insospechadas repercusiones y potenciales beneficios para el desarrollo de niños y niñas, familias y comunidades vulnerables.

\section{Referencias}

Abelenda, J. \& Helfrich, C. (2003). Family resilience and mental illness: The role of occupational therapy. Occupational Therapy in Mental Health, 19(1), 25-39.

Benzies, K. \& Mychasiuk, R. (2008). Fostering family resiliency: A review of the key protective factors. Childeramily Social Work, 14, 103114.

Borges, Z. \& Silva, M. (2010). Promoción de la esperanza y resiliencia familiar. Prácticas apreciativas. Investigación y Educación en Enfermería, 28(2), 250-257.

Bronfenbrenner, U. \& Evans, G. (2000). Developmental science in the $21^{\text {st }}$ century: Emerging questions, theoretical models, research designs and empirical findings. Social Development, 9(1), 115-125.

Colapinto, J. (1995). Dilution of family process in social services: Implications for treatment of neglectful families. Family Process, 34(2), 59-74.

Coletti, M. \& Linares, J. (1997). La intervención sistémica en los servicios sociales ante la familia multiproblemática: la experiencia de Ciutat Vella. Barcelona: Paidós Terapia Familiar.

Cumsille, P. (1996). Problemas y desafíos en la evaluación psicológica de dimensiones familiares. Psykhé, 5, 57-68.

Cyrulnik, B. (2003). El murmullo de los fantasmas. Barcelona: Gedisa.

De Haan, L., Hawley, D. \& Deal, J. (2002). Operationalizing family resilience: A methodological strategy. The American Journal of Family Therapy, 30, 275-291. 
Fonagy, P., \& Target, M. (1997). Attachment and reflective function: Their role in self-organization. Development and Psychopathology, 9, 679-700.

Gómez, E. \& Haz, A.M. (2008). Intervención familiar preventiva en programas colaboradores del Sename: la perspectiva del profesional. Psykhé, 17(2), 53-65.

Gómez, E. \& Cifuentes, B. (2010). Prevención secundaria del maltrato infantil y la negligencia parental: evaluación de resultados del programa "Viviendo en Familia". Manuscrito no publicado.

Gómez, E., Cifuentes, B. \& Ross, M.I. (2010). Previniendo el maltrato infantil: descripción psicosocial de usuarios de programas de intervención breve en Chile. Universitas Psychologica, 9(3), 817833.

Gómez, E., Muñoz, M. \& Haz, A.M. (2007). Familias multiproblemáticas y en riesgo social: características e intervención. Psykhé, 16(2), 4354.

González, C. (2004). Transformación y resiliencia en familias desplazadas por la violencia hacia Bogotá. Revista de Estudios Sociales, 18, 123130.

Gracia, E. \& Musitu, G. (2000). Psicología social de la familia. Barcelona: Paidós.

Greeff, A. \& van der Walt, K.J. (2010). Resilience in families with an autistic child. Education and Training in Autism and Developmental Disabilities, 45(3), 347-355.

Hawley, D. \& De Haan, L. (1996). Towards a definition of family resilience: Integrating individual and family perspectives. Family Process, 35, 283-298.

Hawley, D. (2000). Clinical implications of family resilience. The American Journal of Family Therapy, 28, 101-116.

Johnson, E. (1998). The effect of family functioning and family sense of competence on people with mental illness. Family Relations, 47(4), 443-451.

Kalawski, J.P. \& Haz, A.M. (2003).Y... ¿¿dónde está la resiliencia? Una reflexión conceptual. Revista Interamericana de Psicología, 37(2), 365-372.

Kalil, A. (2003). Family resilience and good child outcomes: A review of the literature. Centre for Social Research and Evaluation, Ministry of Social Development: New Zealand.

Kotliarenco, M. A., Muñoz, M., Gómez, E. \& Armijo, I. (2009). Evaluación pre-post del desarrollo psicomotor y el estilo de apego en los usuarios de los Centros de Desarrollo Infantil Temprano. SUMMA Psicológica UST,6 (2), 89-104. 
Landau, J. (2007). Enhancing resilience: Families and communities as agents for change. Family Process, 46(3), 351-365.

Landau, J. (2010). Communities that care for families: The LINC model for enhancing individual, family, and community resilience. American Journal of Orthopsychiatry, 80(4), 516-524.

Lecannelier, F. (2009). Apego e intersubjetividad. Segunda parte: la teoría del apego. LOM Ediciones: Santiago de Chile.

Lee, M., Greene, G., Hsu, K., Solovey, A., Grove, D., Fraser, J., et al. (2009).Utilizing family strengths and resilience: Integrative family and systems treatment with children and adolescents with severe emotional and behavioral problems. Family Process, 48(3), 395416.

Luthar, S., Cicchetti, D. \& Becker, B. (2000).The construct of resilience: A critical evaluation and guidelines for future work. Child Development, 71(3), 543-562.

Masten, A. \& Obradovic, J. (2006). Competence and resilience in development. Annals of the New York Academy of Sciences, 1094(1), 13-27.

McCartney, K. \& Phillips, D. (2006) (Eds). Blackwell Handbook of Early Childhood Development. Blackwell Publishing: Oxford.

McCubbin, H. \& McCubbin, M. (1988). Typologies of resilient families: Emerging roles of social class and ethnicity. Family Relations, 37, 247-254.

McCubbin, H. \& Patterson, J. (1983). The family stress process: The double ABCX model of family adjustment and adaptation. Marriage and Family Review, 6(1-2), 7-37.

McCubbin, M., Balling, K., Possin, P., Frierdich, S. \& Bryne, B. (2002). Family resilience in childhood cancer. Family Relations, 51(2), 103111.

Minuchin, S. \& Fishman, H.C. (2004). Técnicas de Terapia Familiar. Buenos Aires: Paidós Terapia Familiar.

Navarro Góngora, J. \& Beyebach, M. (1995). Avances en Terapia Familiar Sistémica. Buenos Aires: Paidós Terapia Familiar.

Orthner, D., Jones-Sanpei, H. \& Williamson, S. (2004). The resilience and strengths of low-income families. Family Relations, 53(2), 159167.

Patterson, J. (1988). Families experiencing stress: The family adjustment and adaptation response model. Family Systems Medicine, 5(2), 202237.

Patterson, J. (2002a). Integrating family resilience and family stress theory. Journal of Marriage and Family, 64, 349-360. 
Patterson, J. (2002b). Understanding family resilience. Journal of Clinical Psychology, 58(3), 233-246.

Patterson, J. \& Garwick, A. (1994). Levels of family meaning in family stress theory. Family Process, 3, 287-304.

Petterson, S. \& Burke, A. (2001). Effects of poverty and maternal depression on early child development. Child Development, 72(6), 1794-1813.

Rodrigo, M. J., Camacho, J., Máiquez, M. L., Byrne, S. \& Benito, J. M. (2009). Factores que influyen en el pronóstico de recuperación de las familias en riesgo psicosocial: el papel de la resiliencia del menor. Psicothema, 21(1), 90-96.

Rutter, M. (2007). Resilience, competence and coping. Child Abuse and Neglect, 31, 205-209.

Schade, N., González, A., Beyebach, M. \& Torres, P. (2010). Trastornos somatomorfos en la atención primaria: características psicosociales y resultados de una propuesta de consejería familiar. Revista Chilena de Neuropsiquiatría, 48(1), 20-28.

Shonkoff, J. \& Phillips, D. (Eds) (2000). From Neurons to Neighborhoods: The Science of Early Childhood Development. Washington D.C.: National Academy Press.

Shore, A. (2001). The effects of early relational trauma on right brain development, affect regulation and infant mental health. Infant Mental Health Journal, 22(1-2), 201-269.

Silva, M., Lacharité, C., Silva, P., Lunardi, V. \& Lunardi, W. (2009). Processos que sustentam a Resiliência Familiar: um estudo de caso. Texto \& Contexto Enfermagem, 18(1), 92-99.

Sousa, L. (Ed.). (2008). Strengthening vulnerable families. New York: Nova Science Publishers.

Springer, K., Sheridan, J., Kuo, D. \& Carnes, M. (2007). Long-term physical and mental health consequences of childhood physical abuse: Results from a large population-based sample of men and women. Child Abuse and Neglect, 31, 517-530.

Valencia, E. \& Gómez, E. (2010). Una escala de evaluación familiar eco-sistémica para programas sociales: confiabilidad y validez de la NCFAS en población de alto riesgo psicosocial. Psykhé, 19(1), 89103.

Walker, S., Wachs, T., Gardner, J., Lozoff, B., Wasserman, G., Pollit, E., et al. (2007). Child development: Risk factors for adverse outcomes in developing countries. Lancet, 369, 145-157.

Walsh, F. (2003). Family resilience: A framework for clinical practice. Family Process, 42(1), 1-18. 
Walsh, F. (2004). Resiliencia Familiar: Estrategias para su fortalecimiento. Buenos Aires: Amorrortu Editores.

Walsh, F. (2007). Traumatic loss and mayor disasters: Strengthening family and community resilience. Family Process, 46(2), 207-227.

Werner, E. y Smith, R. (1982). Vulnerable but invincible: A study of resilient children. New York: McGraw-Hill.

Fecha de recepción: 13 de julio de 2010.

Fecha de aceptación: 10 de diciembre de 2010. 
